\title{
Editorials
}

\section{The CCN family of genes: a brief history}

An emerging family of regulatory proteins referred to under the CCN acronym (for Connective tissue growth factor (CTGF), Cystein rich protein (Cyr61), and Nephroblastoma overexpressed gene (nov)) ${ }^{1}$ has been uncovered over the past few years.

The CCN family comprises both positive and negative regulators sharing a common multimodular organisation. New members of the CCN family have been described recently. More are to come.

The chicken CEF10 and homologue murine cyr61 genes were first identified as immediate early genes induced by the pp60 $60^{\mathrm{v}-\mathrm{src}}$ oncogene and serum growth factors, respectively. ${ }^{23}$ The CYR61 protein was shown to promote cell adhesion, migration, and proliferation, probably through potentiating platelet derived growth factor (PDGF) and basic fibroblast growth factor (bFGF) activities. $^{4}$

Human ctgf was also identified as an immediate early gene encoding a connective tissue growth factor $(\mathrm{CTGF})^{5}$ showing mitogenic activity for human umbilac vein endothelial cells (HUVECs) and fibroblasts in culture. ${ }^{6}$

The nov gene was initially characterised as an integration site for the myeloblastosis associated virus (MAV), ${ }^{7}$ which induces kidney tumours that represent a unique model of the Wilms's tumour. ${ }^{8}$ The expression of the nov gene was found to be altered either positively or negatively in human and animal tumours. ${ }^{910}$

The murine Elm1 gene (expressed in low metastatic cells) was reported to be expressed in low metastatic but not in high metastatic K-1735 mouse melanoma cells. It was found to exhibit cell growth inhibitory properties and to suppress the tumorigenic potential of mouse melanoma cells. ${ }^{11}$

Murine rCop-1 gene expression was completely abolished after transformation, and retroviral driven expression of rCop-1 had a dramatic cytotoxic effect on transformed cells, but not on untransformed counterparts. ${ }^{12}$

More recently, three genes involved in the Wnt1 signalling pathway (WISP-1, WISP-2, WISP-3) were shown to be highly related to the CCN family of genes. ${ }^{13}$ The WISP-1 and WISP-2 genes are homologous to Elm1, and rCop-1, respectively.

A novel regulator of osteoblast functions (CTGF-L) was also found to be homologous to CTGF. ${ }^{14}$

The presence of an insulin-like growth factor binding protein (IGFBP)-like motif at the C-terminus of NOVH and the extensive homology of nov, ctgf, cyr61, Elm1, rCOP-1, and IGFBP3 genes at the $5^{\prime}$ end raised the possibility that the proteins might be acting in the IGF signalling pathway. The only evidence is that $\mathrm{CTGF}^{15}$ and $\mathrm{NOV}^{16}$ bind IGF in vitro with a 100-1000 times lower affinity than authentic IGFBPs. Because IGF binding to NOV was not observed under standard ligand blotting assay conditions, ${ }^{17}$ this low affinity binding for IGF remains controversial. No published data suggest binding of CYR61 to IGF. It will be interesting to establish whether the $\mathrm{CCN}$ proteins share common signalling activities with IGFBPs through IGF independent pathways, as recently reviewed for IGFBP-3. ${ }^{18}$

Two motifs sharing identity with the Von Willebrand type C (VWC) domain, probably responsible for oligomerisation, and with the thrombospondin type 1 (TSP1) repeat, responsible for interactions with extracellular matrix proteins, have been recognised in the different members of the CCN family. Although the biological activity of these motifs remains to be established, their conservation in all members of the CCN family argues for their biochemical or structural importance.

The C-terminal module (CT) of the CCN proteins, which was proposed to represent a dimerisation domain, contains a cystine knot motif present and involved in the dimerisation of several growth factors, such as nerve growth factor (NGF), transforming growth factor $\beta 2$ (TGF- $\beta 2$ ), and platelet derived growth factor BB (PBGFBB). It has been established that the C-terminal domain promotes interactions of the nov protein with fibulin $1 \mathrm{C}$ and CTGF. ${ }^{19}$ The absence of this motif in the rCOP-1/CTGF-L/WISP-2 protein ${ }^{12-14}$ might be important.

The multimodular structure of NOV and other CCN proteins raises interesting questions as to the contribution of each individual module to the biological properties of the full length proteins. It is possible that either the biochemical functions of the IGFBP, VWC, TSP, and CT modules contained in these proteins are indeed conserved and sum up in the full length protein, or the presence of each module confers on the whole protein specific biological function(s), which may substitute or add upon those of individual modules.

The bulk of results available to date show that the CCN proteins are involved in the regulation of cell adhesion, migration, proliferation, and survival. The number of studies concerning the role of these proteins in normal key biological processes and in a variety of pathologies is increasing at a steady pace.

Undoubtedly, deciphering the functions of the CCN proteins will result in important progress in understanding the molecular basis of cell growth control and differentiation in normal and pathological conditions.

I am grateful to L Lau for critical reading of the text.

B PERBAL

Laboratoire d'Oncologie Virale et Moléculaire, UFR de Biochimie, Université Paris 7-D. Diderot, 2 Place fussieu, 75005 Paris, France Bernard.Perbal@wanadoo.fr

1 Bork P. The modular architecture of a new family of growth regulators related to connective tissue growth factor. FEBS Lett 1993;327:125-30.

2 Simmons D, Levy D, Yannoni Y, et al. Identification of a phorbol ester-repressible v-w-c- inducible gene. Proc Natl Acad Sci USA 1989;86:1178-82.

3 O'Brien TP, Yang GP, Sanders L, et al. Expression of cyr61, a growth factor-inducible immediate-early gene. Mol Cell Biol 1990;10:3569-77.

factor-inducible immediate-early gene. Mol Cell Biol 1990;10:3569-77.
4 Kireeva ML, Mo FE, Yang GP, et al. Cyr61, a product of a growth factor-inducible immediate-early gene, promotes proliferation, migration, and adhesion. Mol Cell Biol 1996;16:1326-34.

5 Ryseck RP, Macdonald-Bravo H, Mattei MG, et al. Structure, mapping, and expression of fisp-12, a growth factor-inducible gene encoding a secreted cysteine-rich protein. Cell Growth Differ 1991;2:225-33.

6 Bradham DM, Igarashi A, Potter RL, et al. Connective tissue growth factor: a cysteine-rich mitogen secreted by human vascular endothelial cells is related to the SRC-induced immediate early gene product CEF-10. I Cell Biol 1991;114:1285-94.

7 Joliot V, Martinerie C, Dambrine G, et al. Proviral rearrangements and overexpression of a new cellular gene (nov) in myeloblastosis-associated virus type 1-induced nephroblastomas. Mol Cell Biol 1992;12:10-21.

8 Perbal B. Contribution of MAV-1-induced nephroblastoma to the study of Perbal B. Contribution of MAV-1-induced nephroblastoma to the study of
genes involved in human Wilms' tumor development. Crit Rev Oncog 1994; 5enes involved

9 Martinerie C, Huff V, Joubert I, et al. Structural analysis of the human nov proto-oncogene and expression in Wilms tumor. Oncogene 1994;9:272932 . 
10 Martinerie C, Chevalier G, Rauscher F, Jr, et al. Regulation of nov by WT1: a potential role for nov in nephrogenesis. Oncogene 1996;12:1479-92.

1 Hashimoto BY, Shindo-Okada N, Tani M, et al. Expression of the ELM1 gene, a novel gene of the CCN (connective tissue growth factor, cyr61/cef10, and neuroblastoma overexpressed gene) family, suppresses in vivo tumor growth and metastasis of K-1735 murine melanoma cells. $\mathcal{F}$ Exp Med 1998;187:289-96.

12 Zhang R, Averboukh L, Zhu W, et al. Identification of rCop-1, a new member of the CCN protein family, as a negative regulator for cell transformation. Mol Cell Biol 1998;18:6131-41.

13 Pennica D, Swanson TA, Welsh JW, et al. WISP genes are members of the connective tissue growth factor family that are up-regulated in Wnt-1transformed cells and aberrantly expressed in human colon tumors. Proc Natl Acad Sci U S A 1998;95:14717-22.

14 Kumar S, Hand AT, Connor JR, et al. Identification and cloning of a connective tissue growth factor-like cDNA from human osteoblasts encoding a novel regulator of osteoblast functions. F Biol Chem 1999;274:17123-
15 Kim HS, Nagalla SR, Oh Y, et al. Identification of a family of low-affinity insulin-like growth factor binding proteins (IGFBPs): characterisation of connective tissue growth factor as a member of the IGFBP super family. Proc Natl Acad Sci U S A 1997;94:12981-6.

16 Burren C, Wilson E, Hwa V, et al. Binding properties and distribution of insulin-like growth factor binding protein-related protein 3 (IGFBP-rP3/ NovH), an additional member of the IGFBP superfamily. $\mathcal{F}$ Clin Endocrinol Metab 1999;84:1096-103.

17 Chevalier G, Yeger H, Martinerie C, et al. novH: differential expression in developing kidney and a marker of heterotypic differentiation in Wilms' tumor. Am f Pathol 1997;152:1563-75.

18 Oh Y, YamanakaY, Kim H-S, et al. In: Takano K, Hizuka N, Takahashi S-I, eds. Molecular mechanisms to regulate the activities of insulin-like growth factors. Amsterdam: Elsevier, 1998:125-33.

19 Perbal B, Martinerie C, Sainson R, et al. The C-terminal domain of the regulatory protein $\mathrm{NOVH}$ is sufficient to promote interaction with fibulin 1C: a clue for a role of NOVH in cell-adhesion signaling. Proc Natl Acad Sci $U S A$ 1999;96:869-74. 